\section{Incorrect conclusions about unpublished pharmaceutical research}

In a $C M A J$ news article, ${ }^{1}$ David Juurlink states that "there is no obligation on a manufacturer to publish the results of a study," and that he "only sees the evidence that the companies let come to light."

Pharmaceutical companies should not be blamed automatically for the failure to publish an article once a study is complete. Often, these papers are submitted for publication and are rejected by peer-reviewed journals. There are a number of potential reasons why a journal may reject a paper. Peer reviewers may have a bias against a particular drug, company or the entire pharmaceutical industry. Reviewers may think that the quality of the research is inadequate or that there are problems with how the paper is written. In such situations, the paper is usually revised and resubmitted, either to the same journal or to a different one.

Many journals are motivated by impact factor which, from a business standpoint, is completely understandable. Impact factor is a measure of how many times a journal's published articles are cited. ${ }^{2}$ This measure can dictate how good a journal is perceived to be and can lead to increased readership and revenue. This concern over impact factor may lead journal editors to reject a study if they believe that study will not increase their journal's impact factor. I suggest that negative or failed studies are most likely to fall into this category. Thus, a negative study may go unpublished because of a journal's business priorities and not because a pharmaceutical company is withholding data. Unfortunately, this gives the appearance that there is an overrepresentation of positive studies and an underrepresentation of negative studies when the literature is subsequently reviewed.

Any study done in the United States, including most clinical trials with Canadian sites, must be registered with www.clinicaltrials.gov. All pharmaceutical companies must post study results within a reasonable period of time following study completion. Also, some companies require researchers to post study results on public websites. Not adhering to these rules can result in huge financial penalties. Companies risk a decline in stock price if they are accused of not publishing results, and therefore they have much to lose from not publishing even negative studies.

Readers should not assume that nonpublication in a journal automatically equates to the purposeful withholding of data.

\section{James Karagianis MD}

Associate Professor of Psychiatry, University of Toronto, Toronto, Ont., and Psychiatrist in Chief, Waypoint Centre for Mental Health Care, Penetanguishene, Ont.

\section{References}

1. Collier R. UK Parliament calls for sharing of all clinical trial data. CMAJ 2014;186: 176

2. Hecht F, Hecht BK, Sandberg AA. The journal "impact factor": a misnamed, misleading, misused measure. Cancer Genet Cytogenet 1998 Jul 15;104: $77-81$

CMAJ 2014. DOI:10.1503/cmaj.114-0079

\section{Induction of labour}

Mishanina and colleagues ${ }^{1}$ have reported that the risk of cesarean delivery associated with induction of labour was $12 \%$ lower than with expectant management in a systematic review and meta-analysis encompassing 157 randomized controlled trials (RCTs). Although this study presents a widereaching review of trials related to this topic, we are concerned that it sends a message that risks associated with all inductions are equal.

The trials included in the review were highly varied in terms of the indications for induction that were studied. The methods of induction were also not standardized with various methods of pharmacological and mechanical induction carried out. The majority of
RCTs included in the review also contained a mix of both nulliparous and multiparous parturients in their study cohort and individual examination of these groups may have yielded different results.

Furthermore, the range of institutional cesarean delivery rates across the trials included in the study was not addressed, and rates of prelabour cesarean delivery were also not examined.

Settling the argument of induction versus expectant management will be difficult. A large-scale RCT where participants are matched according to parity and indication for induction, and have standardized management for both spontaneous and induced labours is lacking in the literature. The challenges in design and implementation of such a trial are apparent; however, we feel it is misleading to encourage clinicians to apply the findings of the present study to all patients regardless of maternal baseline characteristics and the indication for induction.

Mark P. Hehir MD, Adam Mackie

MBBS, Michael S. Robson MD

National Maternity Hospital (Hehir,

Mackie, Robson), Dublin, Ireland

\section{Reference}

1. Mishanina E, Rogozinska E, Thatthi T, et al. Use of labour induction and risk of cesarean delivery: a systematic review and meta-analysis. CMAJ 2014:186:665-73.

CMAJ 2014. DOI:10.1503/cmaj.114-0077

The most serious outcome of cesarean delivery is maternal death. Landon and colleagues $^{1}$ documented a 1 in 3000 risk of maternal death associated with nonemergency cesarean delivery. Mishanina and colleagues ${ }^{2}$ state that induction had no impact on maternal death. This unqualified statement implies that maternal deaths were reported for all 157 studies and all 31085 study participants. Only 20 of the 157 studies included $(12.7 \%)$ reported occurrences of maternal death. The authors don't show the number of study participants upon which their conclusion about maternal 